Proceedings of the 1997 IEEE

International Conference on Robotics and Automation

Albuquerque, New Mexico - April 1997
(C1987 IEEE. Personal use of this material is permitted. However, permission to reprint/republish this material for advertising or promotional purposes or for creating new collective works for resale or redistribution to servers or lists, or to reuse any copyrighted component of this work in other works must be obtained from the IEEE.

\title{
Design and Comparison of High Strain Shape Memory Alloy Actuators
}

\author{
Alexandra Lu Danny Grant Vincent Hayward \\ Department of Electrical Engineering and \\ Research Centre for Intelligent Machines \\ McGill University \\ Montréal, Québec, H3A 2A7, Canada
}

\begin{abstract}
A simulator is developed to model and design high strain shape memory alloy (SMA) tension actuators. The simulator may be used predict characteristics of a given actuator, or to design its geometry under specifications such as force, speed, stroke and size. The accuracy of the model is verified experimentally in reference to an existing NiTi shape memory alloy prototype actuator. Having developed some confidence in the model, the performance of the proposed actuation mechanism is compared to other existing technologies. In particular, the force-displacement and speed characteristics of a micro-solenoid electro-magnetic actuator and a muscle-size pneumatic actuator are compared to those of the SMA actuators with same dimensions.
\end{abstract}

\section{Introduction}

Shape memory effect (SME) refers to the ability of certain materials to recover a predetermined shape when heated. When a shape memory alloy is in the Martensitic phase (M-phase) at low temperature, it has a very low yield strength and can be deformed quite easily into a new shape, which it retains. However, when the material is heated so that it is in the Parent phase (P-phase), also called Austenitic phase at high temperature, the SMA undergoes a change in crystal structure which causes it to return to its original shape. If the SMA is loaded during this transformation, it can generate large forces. This phenomenon provides an unique mechanism for actuation.

Since the discovery of the shape memory effect, many materials have been found to exhibit such properties. The most common is an alloy of nickel and titanium called Nitinol (NiTi). As an actuator, it is capable of up to $5 \%$ strain recovery and $180 \mathrm{Mpa}$ stress restoration with many cycles [1]. Shape memory alloys have attracted much attention from various fields in industry. Conventional technologies such as electric, hydraulic, and pneumatic actuators have difficulties generating significant forces when their size and weight are scaled down [2]. However, shape memory alloy actuators can attain a high strength to weight ratio which makes them ideal for miniature applications.

While extensive research has been carried out to find practical applications for shape memory alloys, a substantial amount of work has been concerned with establishing mathematical models for these kinds of materials $[3,4]$. Few of these models have been used to design SMA actuators and analyse their performance before they are constructed.

In this paper, we describe a computer software able to model various characteristics such as thermal, material and geometric properties of a type of NiTi shape memory alloy actuator. This simulator is verified by experiments and further used to compare the performance of the proposed SMA actuator with other actuator techniques given similar shape and actuation methods. More specifically, we apply our simulator first to predict the open-loop response of a given actuator, and second to compare high strain shape memory actuators as candidate replacement for electromagnetic and pneumatic actuators.

\section{Design of the Simulator}

The characteristics of a shape memory alloy actuator system can be divided into three groups, namely the thermal property (TP), the material property (MP), and the geometric property (GP). The simulator is designed to model each group and together the overall behaviour of the system. 
The overall system can be described by a constitutive equation

$$
G(T P, M P, G P, \text { current, stress, strain })=0 .
$$

If sufficient input variables to function $\mathrm{G}$ are specified, the relation for the rest of the variables is determined. For a specific SMA actuator, the parameters related to TP, MP and GP are fixed. Therefore, $G=0$ may be used to characterises the input-output relationship of the system.

Most conventional models deal with this relationship since it is important for controller designs. But when designing a specific actuator, stress, strain and some other actuator parameters are given as specifications, while the remaining parameters are to be determined to satisfy the requirements. A particular common version of this problem is to design a SMA actuator given stipulations such as force, stroke, size and material. Conversely, it might be needed to determine desired materials properties such a transition temperatures, in order to achieve a certain level of performance given geometrical constraints.

\section{Modelling the Open-loop Response}

Shape memory alloys exhibit different mechanical properties at different phases. The highly non-linear characteristics of SMA create difficulties for both modelling and control. Most conventional models existing in the literature study the behaviour of SMA from a material science point of view $[5,6]$. A reasonably successful nonlinear model was developed by Ikuta et al. [3] and extended by Madill [4] to account for both major and minor hysteresis behaviour.

In this paper, Ikuta's variable sublayer concept is used to model the phase transformation of a NiTi shape memory alloy, while heating SMA fibers with electric current is used to replace the water bath in Ikuta's experiment. The structure of the model is summarized in the block diagram in Figure 1.

A thermal conduction and heat transfer model is used to predict the temperature-current relationship of SMA in ambient air [4]:

$$
\rho C_{p} V \frac{d T(t)}{d t}=R i^{2}(t)-h A\left(T(t)-T_{\infty}\right)
$$

where $R$ is the resistance of SMA, $\rho$ is the density, $C_{p}$ is the heat capacity, $V$ is the volume of the SMA wire, $h$ is the heat transfer coefficient, $A$ is the surface area of the SMA wire, $T_{\infty}$ is the ambient temperature, and $i$ is the electric current.

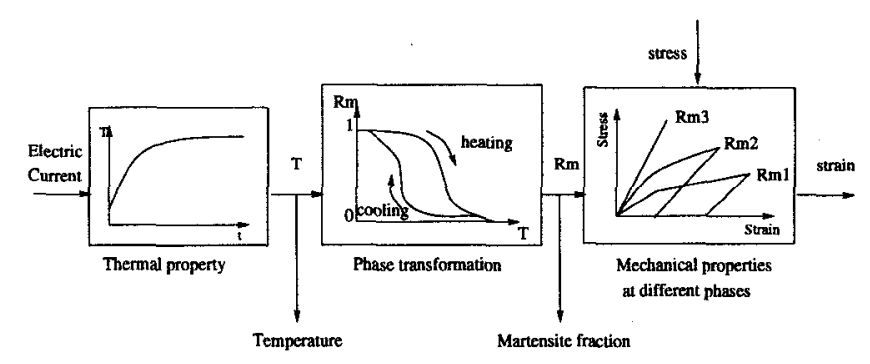

Figure 1: Block Diagram of the SMA Model

Solving equation (2) for $\mathrm{T}$ yields,

$$
T(t)=C e^{-a t} \int_{-\infty}^{t} e^{a \tau} i^{2}(\tau) d \tau+C_{0} e^{-a t}+T_{\infty}
$$

where $C$ and $a$ are thermal constants that can be identified experimentally or calculated from standard heat transfer formulae as follows.

$$
C=\frac{R}{\rho C_{p} V}, \quad a=\frac{h A}{\rho C_{p} V}
$$
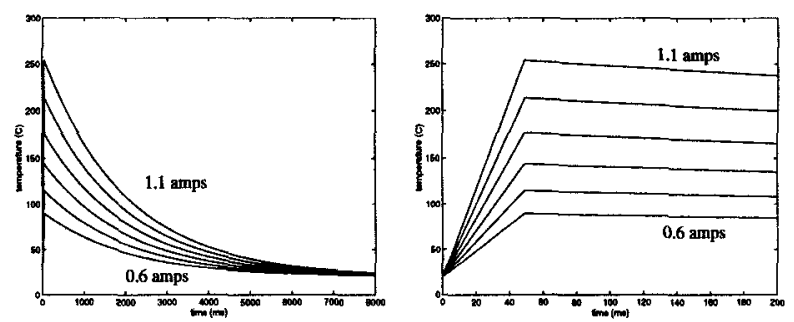

Figure 2: Temperature-current Relationship of SMA

Note that these formulae can only provide approximate estimates, being based on a crude heat transfer model. In practice, these constants are preferably identified experimentally. They provide however very valuable information about their trend when the geometry of the fibers are changed ( $V$ and $A$ ), all other variables being held constant.

Figure 2 illustrates the time domain open loop response to a set of pulse current applied to a shape memory alloy. The duration of the pulse is $50 \mathrm{~ms}$ while the amplitude is varied from $0.6 \mathrm{~A}$ to $1.1 \mathrm{~A}$. The thermal constants $C$ and $a$ calculated based on equation (4) are 0.66 and 4200.0 respectively. Experiments have been set up to identify the Young's modulus of one sample of NiTi shape memory alloy in Martensite and Austenite. The stress-strain curves are obtained when the SMA fiber is cold, or heated with constant 


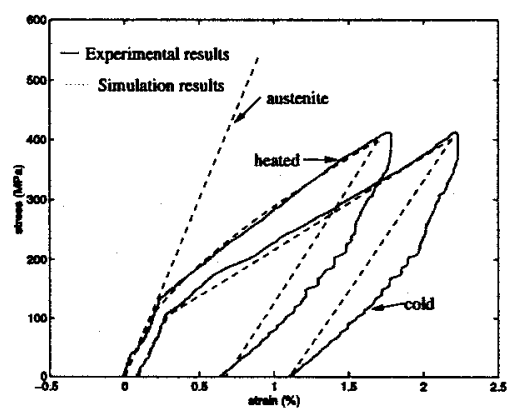

Figure 3: Stress-strain Relationships of SMA at different phases

step current input. Both experimental and simulation results are shown in Figure 3.

\section{Test Cases}

The high strain shape memory alloy actuator consists of a number of thin NiTi fibers woven in a counter rotating helical pattern around supporting disks. It was first proposed by Grant and Hayward [1]. The structure of the actuator is shown in Figure 4. The disks are separated by preloading springs that keep the fibers under tension when relaxed. When the fibers are heated, they contract pulling the disks together.

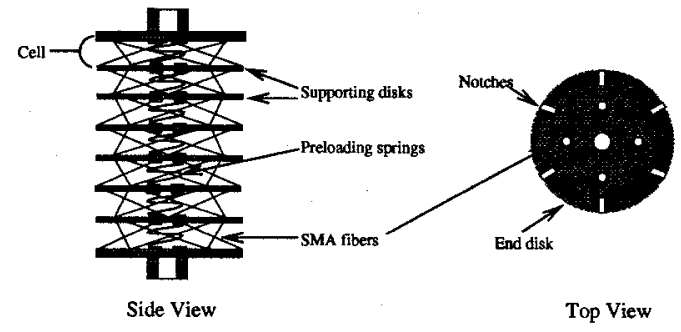

Figure 4: Shape Memory Alloy Actuator

\subsection{Model of the SMA Actuator}

The design parameters involved in modelling the actuator are defined in Figure 5. The forcedisplacement characteristic of the SMA actuator is linked to the stress-strain relationship of the SMA fibers by a geometric factor. The kinematic amplification can be derived by first considering the simplified case consisting of two beams and one fiber as shown
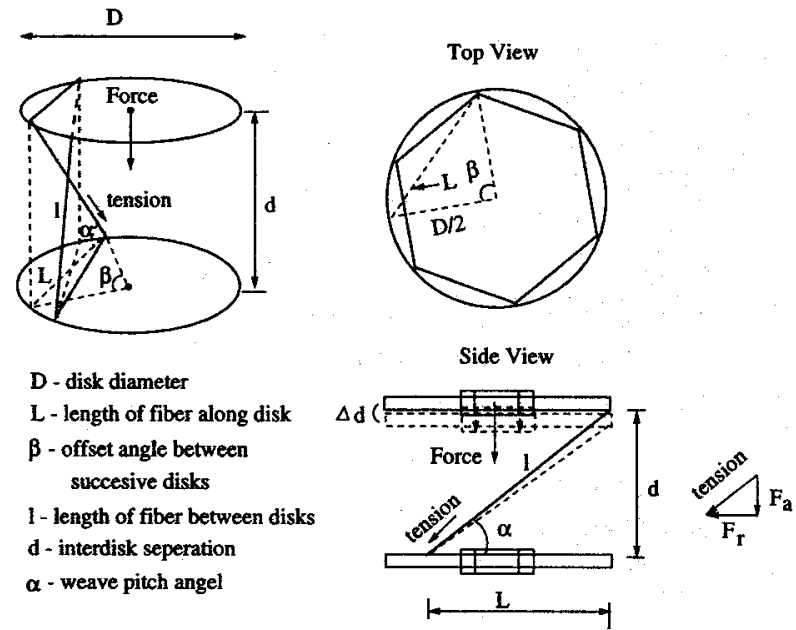

Figure 5: Variables Defined

in the side view in Figure 5. As the fiber contracts, the tension in the fiber can be divided into an axial force $F_{a}$ and a radial force $F_{r}$. Let $t$ be the tension in the fiber, then

$$
F_{a}=-t \cdot \sin (\alpha) .
$$

Since the weave pattern of the actuator is always symmetric, all the radical components of the tension forces of the fibers cancel, leaving only a common axial stress force component $F_{\text {actuator }}$. If the number of notches around the disk is $N_{n}$, there are $2 N_{n}$ fibers in one cell of the actuator. Therefore, the total axial force of the actuator can be expressed as:

$$
F_{\text {actuator }}=-2 N_{n} \sin (\alpha) \cdot t
$$

$2 N_{n} \sin (\alpha)$ is the geometric factor which maps the kinematic gain of the SMA fibers to that of the actuator. The displacement gain, defined as the change in stroke along the separating distance divided by the change in the fiber length is:

$$
\frac{\Delta d}{\Delta l}=\frac{1}{\sin (\alpha)}
$$

The SMA actuator achieves a transformation between force and displacement according to the geometric factor. In this manner, the actuator is no longer limited to the absolute percent strain of the fiber, while the force attenuation can be compensated by using several fibers in parallel.

Equation (6) written in detail is:

$$
\begin{aligned}
& F_{\text {actuator }}=-2 N_{n} \sin (\alpha) \cdot S S\left(\epsilon, R_{m}\right) \cdot s, \\
& \epsilon=\frac{\Delta d \cdot N_{s} \cdot \sin (\alpha)}{\sqrt{l_{a}^{2}+N_{s}^{2} D^{2} \sin ^{2}\left(\frac{\beta}{2}\right)}}
\end{aligned}
$$


where $s$ is the cross section area of the SMA fiber, $R_{m}$ is the Martensite fraction that indicates the phase mix of the actuator, $N_{s}$ is the number of cells, SS represents the phase dependent stress-strain function of the shape memory alloy, $\epsilon$ is the strain of an individual fiber, and $l_{a}$ is the overall length of the actuator. Given the model of the SMA behaviour, the model of the actuator can be constructed based on equation (8) to predict the force-displacement characteristic.

To design an actuator given the desired force, stroke, and dimension, equation (8) has to be solved for the design variables, as a function of given parameters. Numerical methods including Newton and bisection algorithms for finding the roots of non-linear functions are used to determine the design variables when closed-form solutions can not be obtained. For example, the left side of Figure 6 illustrates how to select the length of the actuator according to different strength specifications given a desired stroke, diameter, number of notches and cells. In this particular case, under a certain maximum force, there are two lengths which be be selected for a given required force. The right side of Figure 6 describes how to select the number of disks under similar conditions. The
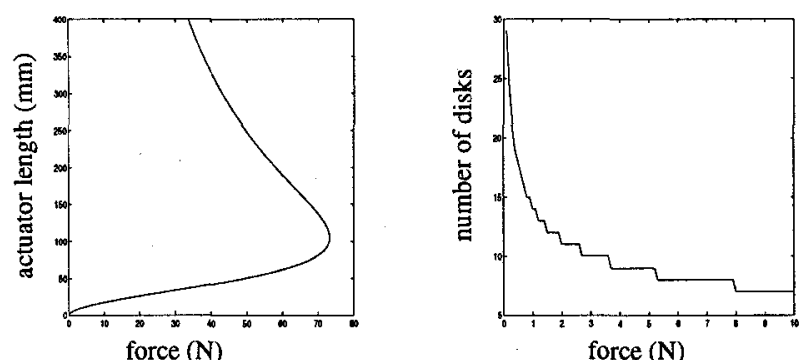

Figure 6: Geometric Property

modelling software can display relationships between any design variables. Of course, it is only convenient to plot relationship between two and sometimes three design variables. Due to its generality, the modelling software could be used by nonlinear search techniques in order to automate the design process.

\subsection{The Prototype Actuator}

The prototype implements only one among many possible configurations of the SMA actuator's geometry. $100 \mu \mathrm{m}$ diameter SMA fibers were chosen for the actuator prototype which was designed to have a desired stroke of $2.5 \mathrm{~mm}$. The actuator consists of 8 disks with 6 notches around each disk. The initial spacing of the disks was set at $2 \mathrm{~mm}$. The actuator constructed is 6 grams in weight and fits inside a 17 $\mathrm{mm}$ diameter cylinder, $30 \mathrm{~mm}$ in length. The maximum pulling force is about $4 \mathrm{~N}$. The simulation of the force-displacement relationship of this specific actuator is shown in Figure 7.

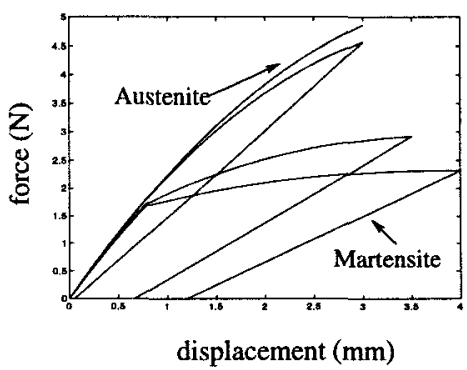

Figure 7: Force-displacement Curves of the Prototype Actuator

During experiments, two SMA actuators are arranged in an antagonistic fashion. The antagonist actuator, being in the cold Martensite phase, produces a bias force which is accounted for in the model.

The experimental time domain open loop response to a set of step current input applied to the actuator is shown in Figure 8. The left side of Figure 8 shows the results of heating one of the actuator with a 50 ms pulse while varying the current amplitude by $.3 \mathrm{~A}$ steps from $4.2 \mathrm{~A}$ to $7.8 \mathrm{~A}$, and the right side of Figure 8 shows the results of heating the actuator with a constant current amplitude while varying the duration of the pulse between $20 \mathrm{~ms}$ and $65 \mathrm{~ms}$. The simulated trajectory curves shown in Figure 9 are obtained by modelling the response of the bias actuator under heating conditions described in the experiments. The thermal constants of the dynamic model are identified from experiments. The simulation result gives good matches. It is easily noticed that the small ripples which are quite apparent on the experimental response are not present in the modelled response. This is easily explained by the fact that the load in the model does not include any inertial term, so second order dynamics are not modelled.

\subsection{A Micro-gripper Actuator}

$\mathrm{Ku}$ et al. [8] developed a remotely controlled microgripper system for microsurgery application. A miniature electromagnetic solenoid actuator was chosen to drive the microgripper. It is an unidirectional device whose force depends on the position of its plunger. Its 

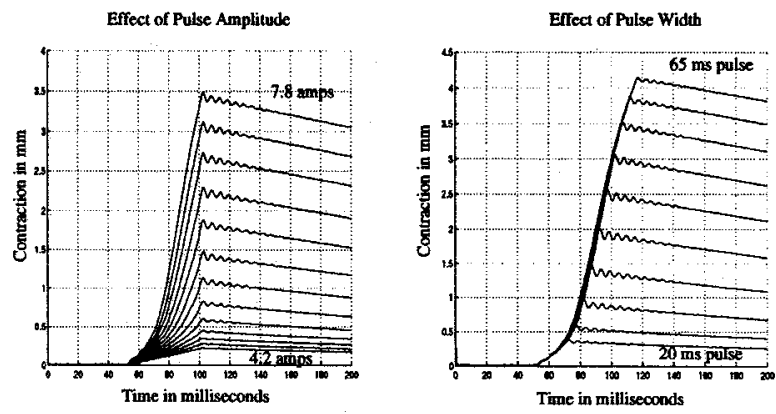

Figure 8: Open Loop Response
Effect of Pulse Amplitude

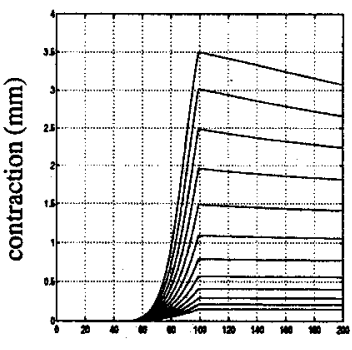

time (ms)
Effect of Pulse Width

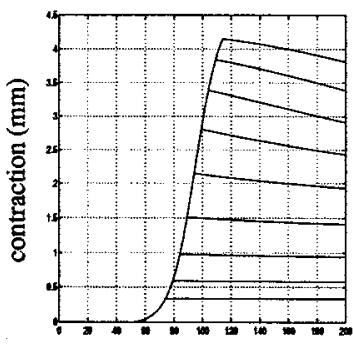

time (ms)
Figure 9: Simulation of the Open Loop Response

force-displacement curve indicates that the maximum pulling force of the solenoid actuator at a displacement of $0.3 \mathrm{~mm}$ is less than $1 \mathrm{~N}$.

We designed a SMA actuator to match the scale of the solenoid actuator used for the microgripper. The diameter and length of the solenoid actuator are estimated to be $6 \mathrm{~mm}$ and $13 \mathrm{~mm}$ respectively. Applying these constraints, two set of configurations were found. The corresponding force-displacement curves predicted are shown in Figure 10. In both cases, 100
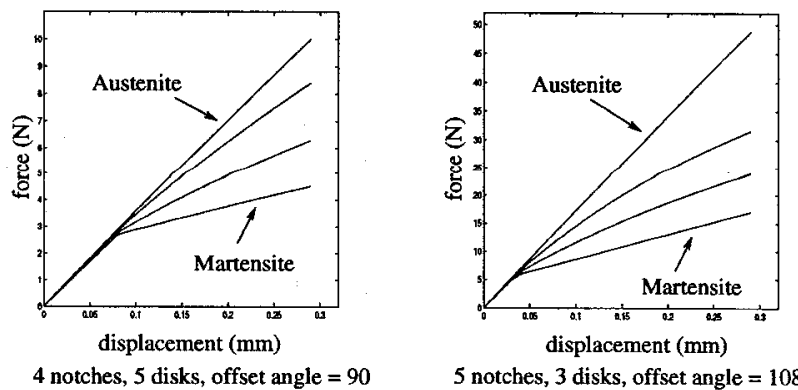

Figure 10: Force-displacement Curves of the Microgripper Actuator $\mu \mathrm{m}$ diameter SMA fibers were chosen for the actuator. The left side of Figure 10 shows the force-displacement characteristic of an SMA actuator with 5 disks, 4 notches, and an offset angle of 90 degrees between successive disks. The pulling force that can be generated by the actuator with a stroke of $0.3 \mathrm{~mm}$ is greater than $5 \mathrm{~N}$ when the fibers are heated with the rated current, while the absolute strain of each fiber is less than $1 \%$. One other configuration of the actuator with same length and diameter has 3 disks, 5 notches, and the offset angle is set to 108 degrees. The strength of the actuator is greater due to more fibers and a smaller displacement gain. As a result, the fibers operate at an absolute strain of about $1.7 \%$ to achieve the 0.3 $\mathrm{mm}$ stroke, yielding a tension greater than $15 \mathrm{~N}$.

The weight of the actuator at such scale would be about 2.5 grams. Clearly, it is much more powerful than the solenoid actuator with same size. In ambient air, the rise time would be about $30 \mathrm{~ms}$. Bathed in a fluid, an order of magnitude of improvement would be expected.

\subsection{An Artificial Muscle Actuator}

The McKibben artificial muscle pneumatic actuator reported by Chou et al [9] consists of an internal bladder surrounded by a braided mesh shell. When the internal bladder is pressurized, the actuator shortens and produces tension if it is coupled to a mechanical load. The Bridestone McKibben actuator tested in [9] is $147 \mathrm{~mm}$ in length and $15 \mathrm{~mm}$ in diameter. The tension indicated from the experimental result at 4 bar pressure and $25 \mathrm{~mm}$ displacement is about $50 \mathrm{~N}$. The maximum tension reported for this type of actuator is $260 \mathrm{~N}$.

For the same length and diameter specified above, a SMA actuator could have 24 notches (48 fibers in parallel), 35 disks, and an offset angle of 90 degrees. The simulation of the force-displacement curves of the actuators using $100 \mu \mathrm{m}$ and $200 \mu \mathrm{m}$ diameter SMA fibers respectively are shown in Figure 11. The displacement gain is 3.4. The fibers in both actuators operate at $2 \%$ absolute strain to have a $25 \mathrm{~mm}$ stroke. The maximum tension of the actuator using $100 \mu \mathrm{m}$ and $200 \mu \mathrm{m}$ diameter SMA fibers are $38 \mathrm{~N}$ and $150 \mathrm{~N}$ respectively, with rated heating current. The weight of an actuator is about 16 to 20 grams. Using 200 $\mu \mathrm{m}$ diameter fibers results in a much stronger actuator, but suffering from a reduced response time. We would expect the rise time an actuator with the thicker fibers to be $60 \mathrm{~ms}$. The Bridgestone actuator chosen from [9] for comparison yields the highest tension and stiffness intensities among all the pneumatic actua- 
$100 \mu \mathrm{m}$ diameter fiber

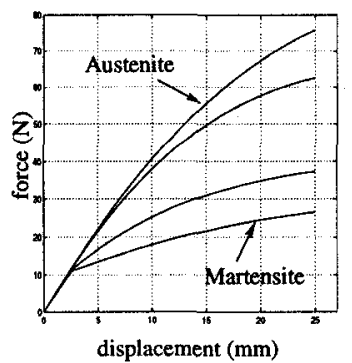

$200 \mu \mathrm{m}$ diameter fiber

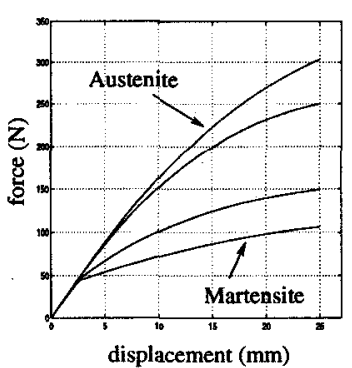

and Robotics" (MSA-4), and an operating grant from NSERC, the National Science and Engineering Council of Canada.

\section{References}

[1] D. Grant and V. Hayward, Design of shape memory alloy actuator with high strain and variable structure control, Procs. of IEEE International Conference on Robotics and Automation, volume 3, pages 2305-2312, 1995.

Figure 11: Force-displacement Curves of the Muscle Actuator

tors reported in that paper. The maximum tensions for the McKibben actuators using nylon shell and fibreglass shell are $110 \mathrm{~N}$ and $56 \mathrm{~N}$ respectively. SMA actuators for a same size package would yield more strength than any of these designs. They also would be much simpler to manufacture and would not require servo valves. Experiments with smaller actuators using $100 \mu \mathrm{m}$ fibers (fiber diameter is the dominating factor) indicate much wider dynamic range and speed ranges.

\section{Conclusions}

A software simulator for shape memory alloy actuator systems is constructed to predict the currentstress-strain dynamics of a NiTi shape memory alloy high-strain actuators. This simulator is also used to design geometries of the proposed SMA actuator under specifications such as force, stroke, size, and speed.

The performance of such an actuator is compared with that of a miniature solenoid actuator and a pneumatic muscle size actuator under similar dimensions. The SMA actuator is superior to the solenoid actuator in power, weight and displacement range. The comparison of the SMA actuators with the Bridgestone artificial muscle indicates that shape memory alloy actuators can also be employed in larger structures.

\section{Acknowledgements}

The research was mostly funded by IRIS (Phase II), the Institute for Robotics and Intelligent Systems part of Canada's National Centers of Excellence program (NCE), under the heading "Machine Sensing and Actuation: Computational Sensing for Vision
[2] K. Ikuta, Micro/miniature shape memory alloy actuator, Procs. of IEEE International Conference on Robotics and Automation, Cincinnati, pages 2156-2161, 1990

[3] K. Ikuta, M. Tsukamoto and S. Hirose, Mathematical model and experimental verification of shape memory alloy for designing micro actuator, Procs. of IEEE MicroElectro Mechanical Systems Conference, pages 103-108, 1991.

[4] D.R. Madill, Modelling and stability of a shape memory alloy position control system, Master's thesis, Applied Science, University of Waterloo, 1993.

[5] C. Liang and C.A. Rogers, A multi-dimensional constitutive model for shape memory alloys, Journal of Engineering Mathematics 26: 429-443, 1992.

[6] T.E. Buchheit, S.L. Kumpf and J.A. Wert, Modelling the stress-induced transformation behaviour of shape memory alloy single crystals, Acta metall. mater. Volume 43, No. 11, pages 4189-4199, 1995.

[7] D. Grant, Shape memory alloy actuator with an application to a robotic eye, Master's thesis, McGill University, 1995.

[8] S. Ku and S.E. Salcudean, Design and control of a teleoperated microgripper for microsurgery, Procs. of IEEE International Conference on Robotics and Automation, pages 889-894, 1996.

[9] C.P. Chou and B. Hannaford, Measurement and modelling of McKibben pneumatic artificial muscles, IEEE Trancs on Robotics and Automation, volume 12, no. 1, pages 90-102, 1996. 